\title{
Relation between Immune cell response and stemness genes expression in breast cancer; A new approach in NANOG gene and Let7-a expression in breast cancer cell lines
}

\author{
Zeynab Aliyari-Serej ${ }^{1,2,3}{ }^{\circledR}$, Ayyub Ebrahimi ${ }^{4}{ }^{\circledR}$, Tohid Kazemi ${ }^{2,5}{ }^{\circledR}$, Souzan Najafi ${ }^{2}$, Elmira Roshani' ${ }^{2}$, Mohammad Ebrahimi- \\ Kalan $^{6}{ }^{\circledR}$, Behzad Baradaran ${ }^{2,5 *}$ \\ ${ }^{1}$ Department of Applied Cell Sciences, School of Advanced Medical Sciences, Tabriz University of Medical Sciences, Tabriz, Iran \\ ${ }^{21}$ mmunology Research Center, Tabriz University of Medical Sciences, Tabriz, Iran \\ ${ }^{3}$ Student Research Committee, Tabriz University of Medical Sciences, Tabriz, Iran \\ ${ }^{4}$ Department of Molecular Biology and Genetics, Faculty of Arts and Sciences, Halic Uuniversity, Istanbul, Turkey \\ ${ }^{5}$ Department of Immunology, Faculty of Medicine, Tabriz University of Medical Sciences, Tabriz, Iran \\ ${ }^{6}$ Department of Epidemiology, Stempel College of Public Health, Florida International University, Miami, FL, USA
}

\section{*Correspondence to}

Behzad Baradaran, Email:

behzad_im@yahoo.com ,

baradaranb@tbzmed.ac.ir

Received 20 Dec. 2019

Accepted 7 Jan. 2020

Published online 6 May 2020

Keywords: Breast cancer Immunologic response, Stemness, Cancer stem cells, Novel cancer therapy
Citation: Aliyari-Serej Z, Ebrahimi A, Kazem T, Najafi S, Roshani E, Ebrahimi-Kalan M, et al. Relation between Immune cell response and stemness genes expression in breast cancer; A new approach in NANOG gene and Let7-a expression in breast cancer cell lines. Immunopathol Persa. 2020;6(2):e21. DOI:10.34172/ ipp.2020.21.

\begin{abstract}
Introduction: Failure and recurrence in breast cancer treatment cause a great obstacle in cancer therapy and identification of cell population named cancer stem cells (CSCs) in the tumor can be led us to define it as target in novel therapeutic strategy.

Objectives: The aim of this study is the finding of correlation between stemness and metastatic characteristic, also knowing CSCs as a potential target of therapy because of its developmental behavior and similarities with normal stem cells.

Materials and Methods: Here, we focus on the expression of NANOG in breast CSCs, a key molecule in the physiological process of stem cells and the Let-7a that is involved in the differentiation of the cells.

Results: In this work, we found that NANOG was highly expressed in SKBR3 and down-regulation of let-7a, as a differentiation miRNA, was found in MDA-MB-468 cells.

Conclusion: It will be critical for the developing of effective anti-tumor drugs, utilizing mentioned concepts Inhibition of NANOG in combination with Let-7a up-regulation can help to decrease the stemness and increase the differentiation of CSCs. The decrease of stemness and increase of differentiation initiate the apoptotic process. So, modification in the mechanism of apoptosis beside anti-cancer drugs provide a good preclinical study goal However, in order to these drugs become clinical, the problems of their side effects and toxicity must be solved. Differentiation of CSCs provides an optimal condition to activity of immune cells which never let them escape from immune cells by alteration of immunogenicity.
\end{abstract}

Introduction

One of the most prevalent malignancies among US females is breast cancer (BC) and also it is as the $2^{\text {nd }}$ cause of cancerassociated deaths in the world (1). That is a heterogeneous disease, associated with distinctive histological patterns and different biological features and clinical behaviors (2). $\mathrm{BC}$ has several intrinsic molecular subtypes that were classified into; luminal A, luminal $\mathrm{B}$, human epidermal growth factor receptor 2 overexpressing (HER2-OE), and basallike tumors. These subtypes show highly differences in prediction of overall survival and response to therapy $(3,4)$. However, the relapse and high rate of mortality remain as big problems a few years later after therapy.

\section{Key point}

In this study we evaluate the expression of the NANOC and Let-7a in the breast cancer cell lines. SKBR3 cells express a high level of NANOG and low level of Let-7a. So, the inhibition of NANOG and increasing the Let-7a expression can reduce the stemness in breast cancer and helps to improve the breast cancer therapy.

The failure and recurrence in $\mathrm{BC}$ treatment is due to presence of cell population which are resistant to therapy and metastasize to distant organs. This population displays stem cell properties.

The origin of breast cancer stem cells (CSCs) is the mammary multipotent stem cells with genetic defects that affect differentiation and

\footnotetext{
Copyright (c) 2020 The Author(s); Published by Nickan Research Institute. This is an open-access article distributed under the terms of the Creative Commons Attribution License (http://creativecommons.org/licenses/by/4.0), which permits unrestricted use, distribution, and reproduction in any medium, provided the original work is properly cited.
} 
self-renewal related pathways (5). The source of these cells is important for prevention, early detection, and treatment of BC (6). There are similarities between CSCs and normal stem cells. They are multipotent, quiescent, with ability to self-renew and helps to recovery of the tumor (7). Many signaling pathways are involve in regulation of normal stem cell development and self-renewal and disturbing of these pathways has been observed in cancer (8). Thus, targeting key signaling cascades in cancer cells can be an efficient way to improve the prognosis in BC therapy and overcome to therapeutic failure (9). One of the important stemness factors is the NANOG that is involved in the maintenance of pluripotency and self-renewal of CSC $(10,11)$. NANOG expression was investigated in several types of cancer, including $\mathrm{BC}$ and it is related to a poor prognosis for $\mathrm{BC}$ patients (12-14). In addition of stemness factors, miRNAs are important factors in regulating of CSC properties like pluripotency, invasion, EMT and migration, which are involve in metastasis of tumor $(15,16)$. One of these miRNAs is Let-7 that regulates the CSCs specifications and its overexpression decreases the tumor metastasis and also the rate of cell proliferation (17-19).

Beside of very clear role of CSCs in proliferation, metastasis and therapeutic resistance of tumor cells (20), more recently the role of CSCs in the extent of tumor through diminishing of immune cells surveillance intratumor microenvironment has been investigated (21). Additionally, CSCs are potent to escape from immune cells by alteration of immunogenicity (22). Furthermore, experimental model showed CSCs directly related to progression of tumor cells and can be useful if we destroyed or ablated those (23). Recently studies focus on the modulation of immune cells functions by CSCs microenvironment. Chronic inflammatory condition is a key factor in the tumor microenvironment that leads to proliferation and metastasis of the cancerous cells through the immunosuppression of immune cells like T cytotoxic and Natural killer cells (24-26).

In this study we evaluated the expression of stemness factor named NANOG and differentiation miRNA known as Let-7a in BC cell lines (SKBR3, MCF7, MDA231 and MDA468).

\section{Objectives}

The purpose of this study was to understand the relation between differentiation and stemness. We hypothesized that the high level of NANOG should be with low level of the Let-7a.

\section{Materials and Methods \\ Cell lines}

The human BC cell lines (MCF-7, SK-BR-3, MDA-MB-231, and MDA-MB-468) were purchased from Immunology Research Center of Tabriz University of Medical Sciences. These cell lines were maintained in Roswell Park Memorial Institute 1640 (RPMI1640) (Gibco; Thermo Fisher,
Waltham, USA) containing 10\% fetal bovine serum (FBS) (Gibco), 1\% antibiotics (penicillin/streptomycin) at $37 \mathrm{C}$ in a water-saturated atmosphere of 5\% CO2.

\section{Real-time quantitative PCR analysis for $m R N A$ relative quantification}

Trizol reagent (GeneAll') was used to extraction of total RNA from cells, and the reverse transcription reactions were done with random hexamer primer and a Reverse Transcriptase M-MLV (Biofact) according to the manufacturer's protocol. For miRNA (Let-7a) detection, complementary DNA (cDNA) samples were prepared by Universal cDNA Synthesis Kit, by using 100 ng total RNA in a reaction volume of $15 \mu \mathrm{L}$ by using the protocol of cDNA synthesis Kit (BONmiR).

The expression levels of all miRNAs and mRNAs in each cell line were assessed using SYBR green-based quantitative reverse transcriptase real-time polymerase chain reaction (qRT-PCR) (BioFact). We used U6 as an internal control to normalize the miRNAs level expression and 18s (for cell lines SK-BR-3, MCF-7, MDA-MB-468, and MDA-MB-231). Amplification was done as follows: $95^{\circ} \mathrm{C}$ for 10 minutes, 40 cycles of $95^{\circ} \mathrm{C}$ for $10 \mathrm{~s}$ and $60^{\circ} \mathrm{C}$ for 10 seconds. The primer sequences have been shown in Table 1. All of the samples have been done in triplicate. The $2^{-\Delta \Delta \mathrm{Ct}}$ method was used to determine the relative quantitation of gene expression levels. The sequences of primers used and annealing temperatures are listed in Table 1.

\section{Western blot analysis}

Western blot has been used to identify a specific protein. Here we worked on the protein mixture based on separation by molecular weight via electrophoresis gel. Then the protein bands transferred to western blotting membrane and incubated with antibodies.

The cold PBS was used to wash the cells and then the cells were lysed using ice cold radio immunoprecipitation assay (RIPA) buffer (1 mM EDTA with pH 8, $150 \mathrm{mM}$ $\mathrm{NaCl}, 1 \%$ Triton $\mathrm{X}-100,1 \%$ SDS, $50 \mathrm{mM}$ Tris- $\mathrm{HCl}$ with $\mathrm{pH}$ 7.4) containing protease inhibitor cocktail (PMSF: 1 $\mu \mathrm{L}$ per well, protease inhibitor: $1 \mu \mathrm{L}$ per well, phosphatase inhibitor: $1 \mu \mathrm{L}$ per well) (Roche Diagnostics $\mathrm{GmbH}$ ) for 30 min. Before centrifugation, the cells were vortexed for 50 seconds, and this step was repeated five times. Then, cell suspension was centrifuged at $14000 \mathrm{rpm}$ for 10 minutes

Table 1. The PCR primer sequences

\begin{tabular}{ll}
\hline Target gene & Primer sequence \\
\hline Let-7a-5p & 5'UGAGGUAGUAGGUUGUAUAGUU3' \\
NANOG & F: 5'TTTGTGGGCCTGAAGAAAACT3' \\
& R: 5'AGGGCTGTCCTGAATAAGCAG3' \\
18s & F: 5'GCTTAATTTGACTCAACACGGGA3' \\
U6 & R: 5'AGCTATCAATCTGTCAATCCTGTC3' \\
\hline
\end{tabular}


at $4^{\circ} \mathrm{C}$. Finally, cellular debris was outed.

In this step, we have determined the volume of protein extract to ensure $50 \mu \mathrm{g}$ in each well. $5 \mu \mathrm{L}$ sample buffer was added to the sample, and the volume was making in each lane equalized using double distilled $\mathrm{H} 2 \mathrm{O}$ (dd H2O). The well was mixed and was heated the samples with dry plate for 5 minutes at $59^{\circ} \mathrm{C}$. $12.5 \%$ SDS-polyacrylamide gel electrophoresis was used to isolate the proteins bands were transferred to polyvinylidene difluoride membranes (Roche Diagnostics GmbH). We used the 0.5\% Tween-20 in PBS/Tween-20 $\left(0.05 \%\right.$, v/v) overnight at $4^{\circ} \mathrm{C}$ to blocking. In the next step, the membrane incubated in the polyclonal primary antibodies against NANOG and monoclonal antibody against $\beta$-actin in 1 hour at room temperature. The washing buffer containing $0.05 \%$ Tween-20 and PBS was used to wash the membrane for four 10 minutes and after washing the membrane was incubated with secondary antibody named horse radish peroxidase-linked rabbit anti-goat and rabbit anti-mouse antibody (Razi Institute, Tehran, Iran) for 1 hour at room temperature. Next the membrane was washed again and the protein bands were detected by BM chemiluminescence blotting substrate POD (Roche Diagnostics GmbH, Mannheim, Germany) and the light signals detected by digital imaging.

\section{Ethics issues}

This study was approved by the Ethics Committee of the Tabriz University of Medical Sciences (IR.TBZMED.VCR. REC.1397.255). All procedures performed in accordance with the ethical standards of the Tabriz University of Medical Sciences. This study was extracted from a research proposal of Immunology Research Center and School of Advanced Medical Sciences at this university.

\section{Statistical analysis}

Statistical analyses were processed by the GraphPad Prism v. 6.0 software. All data were analyzed by using the independent $t$ test and one-way analysis of variance (ANOVA). $P$ values $<0.05$ were considered to be statistically significant.

\section{Results}

The NANOG $m R N A$ and protein levels in BC cell lines

Previous studies reported that NANOG was required for selfrenewal and pluripotency of CSCs, suggesting the important functions of NANOG in stem cell biology. To detect whether NANOG is highly expressed in breast CSCs, we used qPCR and western blot methods. As shown in Figures 1a and b, expressions of NANOG mRNA and protein levels were both obviously higher in SKBR3 cells compared to other cell lines. The level of NANOG was 66-fold higher in SKBR3 cells compared to control cells (Figure 1a). The mRNA levels of these genes moderately increased in MCF-7, MDAMB-231 and marginally in MDA-MB-468 cells. We used fibroblast cells as negative control (Figure 1a).

Here we evaluated the levels of NANOG protein using immunoblot analysis. That way, the proteins were isolated
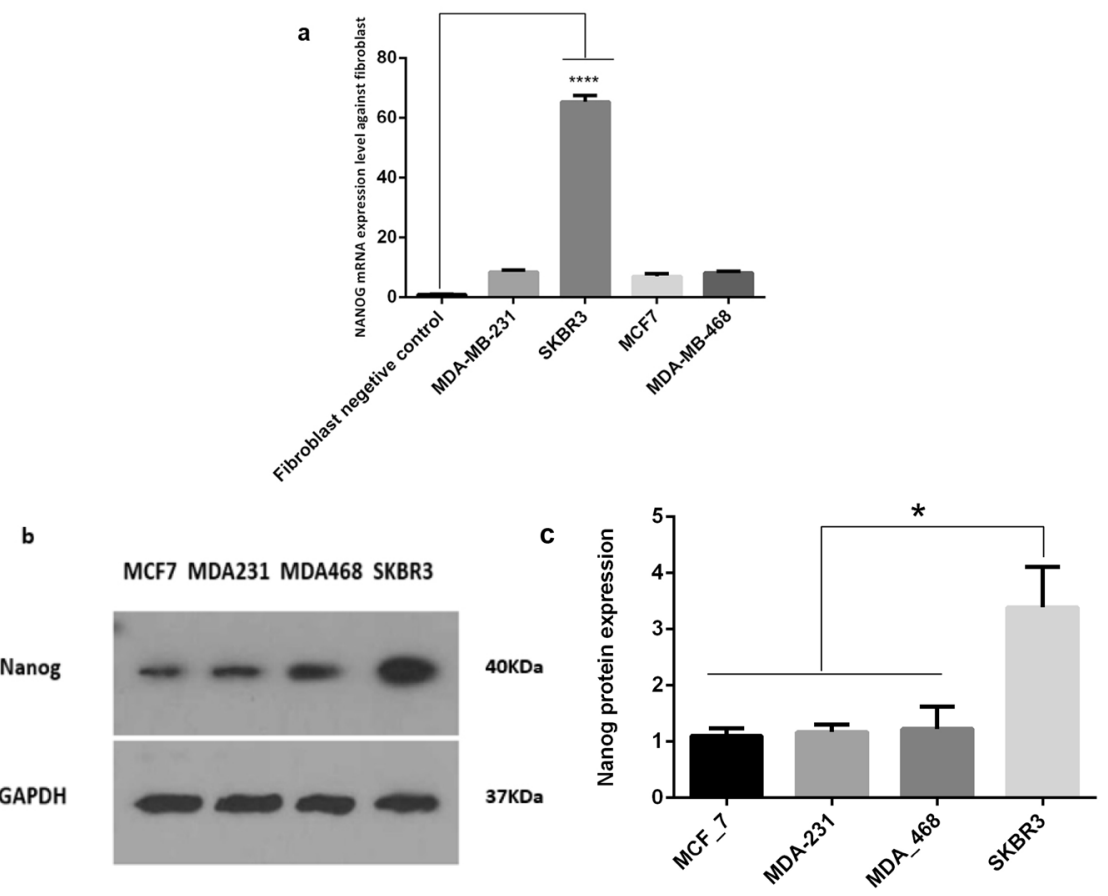

Figure 1. NANOG was highly expressed in breast CSCs-enriched population. (a) Expression of NANOG mRNA in BC cells lines. The total RNA was extracted and after reverse transcription, expression of NANOG mRNA was determined by real-time PCR. (b) Expression of NANOG protein in BC cells lines. The total protein was extracted and expression of NANOG protein was analyzed by Western blot assay. Data are reported as means \pm SD of three separate experiments, **** indicate $P \leq 0.0001$ and * indicate $P \leq .0 .05$. 
from the $\mathrm{BC}$ cell lines. Our data showed that in comparison with the other BC cell lines, the SKBR3 cells have higher expression levels of NANOG protein (Figure 1b).

\section{Let-7a expression in breast cancer cell lines}

According to previous studies, Let-7a expresses in differentiated cells and mature tissue, but is not expresses in the undifferentiated embryonic cells and it was found as a tumor suppressor in numerous cancers types such as BC (27).

To evaluate the level of Let-7a miRNA in BC cell lines, we used real-time PCR method. Our results indicated that the level of Let-7a was in contrast to the stemness factor NANOG. So, the level of Let-7a was significantly higher in MDA-468 than other cell lines (Figure 2).

\section{Discussion}

$\mathrm{BC}$ is a malignancy with histological, molecular and epidemiological heterogeneity. In fact, this heterogeneity causes a big challenge to the development of effective cancer therapeutics. Failure and recurrence in BC treatment cause a great obstacle in cancer therapy (28). Pre-clinical studies have provided evidence that population with stem cell properties were found in tumors which termed as CSCs (29). The concept of CSC underscores that the small proportion of cancer stem-like cells may drive tumor growth, metastasis and resistance to conventional cancer therapy, thereby having profound clinical implications for cancer therapeutics. The origin of breast CSCs is the mammary multipotent stem cells with genetic defects that affect pathways related to self-renewal and differentiation (30). The lack of efficacy of current cancer therapeutics particularly in advanced and metastatic disease requires novel strategies that can specifically target CSC populations. Decreasing in tumor size and inhibition of its growth never show the CSCs percentage diminishing (31).

Until now there are numbered characteristics of some of the most important molecules involved in developmental biology and abnormal issue in the body. However, in

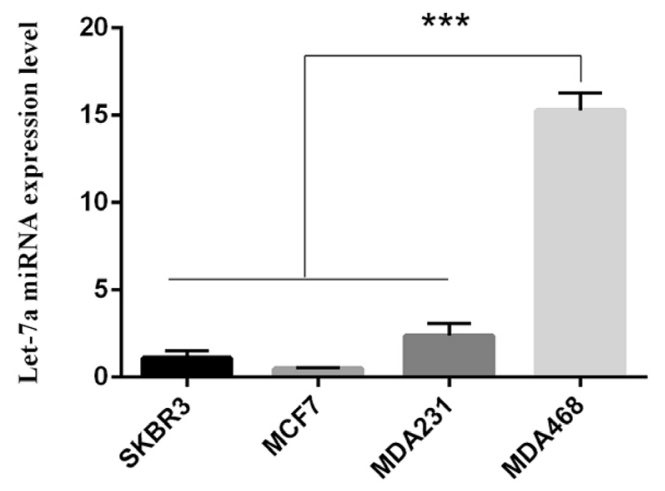

Figure 2. Let-7a was lowly expressed in BC cell lines. (a) Expression of. Let7a miRNA in BC cells lines. The total RNA was extracted and after reverse transcription, expression of Let-7a mRNA was determined by real-time PCR. Data are reported as means \pm SD of three separate experiments, ${ }^{* * * *}$ indicate $P \leq 0.0001$. continue, we showed another essential molecule that affected the growth of tumor cells passively or negatively.

Here, we focus on the expression of NANOG in breast CSCs, a key molecule in the physiological process of stem cells. In this work, we found that NANOG was highly expressed in SKBR3 (Figure 1). Down- regulation of let-7a was found in MDA-MB-468 cells.

Our results showed that there was an invert relation between NANOG and Let-7a. Thus, we can understand that dysregulation of NANOG and Let-7a are very important in cancer trigger and progression of $\mathrm{BC}$ through the apoptosis process induction or differentiation of them. We can use these results in next studies. For example, the inhibition of NANOG in combination with Let-7a upregulation can help to decrease the stemness and increase the differentiation of CSCs. According to previous studies we know that the decrease of stemness and increase of differentiation cause to initiation of apoptosis. Thus, anti cancer drugs beside increase in apoptosis of tumoral cells can be as a interesting preclinical study. On the other hand, CSCs are potent to escape from immune cells by alteration of immunogenicity. CSCs delayed or cut the response of immune cells by secretion of immunosuppressive factors, mimicking of antigen-presenting cells. A powerful understanding of the cell signaling pathways in CSCs differentiation inducer agents can be led us to define and control treatment problems such as metastasis and chemoresistance. Indeed, knowledge about stem cells and particularly CSCs can be led us to define a novel therapeutic strategy and CSCs interventions.

\section{Conclusion}

In this work, we found that NANOG was highly expressed in SKBR3 and and Let-7a was downregulated in MDAMB-468 cells. It will be critical for the developing of effective anti-tumor drugs, utilizing mentioned concepts. Our result showed that metastasis and chemoresistance of cancer have a direct relation with stemness characteristic of cancers. Differentiation of CSCs provides an optimal condition to activity of immune cells which never let them escape from immune cells by alteration of immunogenicity.

\section{Authors' contribution}

$B B, A E$, and ZAS designed the study. ZAS, SN, ER, BB performed the experiments. ZAS, BB, AE, and TK analyzed the data. ZAS, MEK, AE and $B B$ wrote the manuscript. All authors approved the final version of the manuscript.

Conflicts of interest

The authors declare no conflicts of interest.

Ethical considerations

Ethical issues (including plagiarism, data fabrication, double publication) have been completely observed by the authors.

Funding/Support

This study was supported by Tabriz University of Medical Sciences (Grant N0. 59955). 


\section{References}

1. Siegel RL, Miller KD, Fedewa SA, Ahnen DJ, Meester RGS, Barzi A, et al. Colorectal cancer statistics, 2017. CA Cancer J Clin. 2017;67(3):177-193. doi: 10.3322/caac.21395.

2. Weigelt B, Reis-Filho JS. Histological and molecular types of breast cancer: is there a unifying taxonomy? Nat Rev Clin Oncol. 2009;6:718-30. doi: 10.1038/nrclinonc.2009.166.

3. Perou CM, Sørlie T, Eisen MB, van de Rijn M, Jeffrey SS, Rees $\mathrm{CA}$, et al. Molecular portraits of human breast tumours. Nature. 2000;406:747-52. doi: 10.1038/35021093

4. Sorlie T, Tibshirani R, Parker J, Hastie T, Marron JS, Nobel A, Deng $S$, et al. Repeated observation of breast tumor subtypes in independent gene expression data sets. Proc Natl Acad Sci U S A. 2003;100:8418-8423. doi: 10.1073/pnas.0932692100

5. Klonisch T, Wiechec E, Hombach-Klonisch S. Cancer stem cell markers in common cancers-therapeutic implications. Trends Mol Med. 2008 Oct;14:450-60. doi: 10.1016/j.molmed.

6. Reya T, Morrison SJ, Clarke MF, Weissman IL. Stem cells, cancer, and cancer stem cells. Nature. 2001;414:105. doi: 10.1038/35102167

7. Kim YJ, Siegler EL, Siriwon N, Wang P. Therapeutic strategies for targeting cancer stem cells. Mol Ther. 2017;25:2607-2619. doi: 10.1016/j.ymthe.2017.08.010.

8. Phillips TM, McBride WH, Pajonk F. The response of CD24-/ low/CD44+ breast cancer-initiating cells to radiation. J Natl Cancer Inst. 2006;98:1777-1785.doi: 10.1093/jnci/djj495

9. Wang J, Zheng Y, Zhao M. Exosome-based cancer therapy: implication for targeting cancer stem cells. Front Pharmacol. 2017 Jan 12;7:533. doi: 10.3389/fphar.2016.00533.

10. Cavaleri F, Schöler HR. Nanog: a new recruit to the embryonic stem cell orchestra. Cell. 2003;113:551-552. doi: 10.1016/ s0092-8674(03)00394-5

11. Das B, Pal B, Bhuyan R, et al. MYC Regulates the HIF2alpha Stemness Pathway via Nanog and Sox2 to Maintain SelfRenewal in Cancer Stem Cells versus Non-Stem Cancer Cells. Cancer Res. 2019;79(16):4015-4025.

12. Kondo T, Setoguchi T, Taga T. Persistence of a small subpopulation of cancer stem-like cells in the C6 glioma cell line. Proc Natl Acad Sci U S A. 2004;101:781-786.doi: 10.1073/pnas.0307618100

13. Ling G-Q, Chen D-B, Wang B-Q, Zhang L-S. Expression of the pluripotency markers Oct3/4, Nanog and Sox2 in human breast cancer cell lines. Oncol Lett. 2012;4:1264-1268. doi: 10.3892/ol.2012.916

14. Han J, Zhang F, Yu M, Zhao P, Ji W, Zhang H, et al. RNA interference-mediated silencing of NANOG reduces cell proliferation and induces G0/G1 cell cycle arrest in breast cancer cells. Cancer Lett. 2012;321:80-8. doi: 10.1016/j. canlet.2012.02.021

15. Wang W, Luo Y-p. MicroRNAs in breast cancer: oncogene and tumor suppressors with clinical potential. J Zhejiang Univ Sci B. 2015:16:18-31. doi: 10.1631/jzus.

16. Melo SA, Esteller M. Dysregulation of microRNAs in cancer: playing with fire. FEBS Lett. 2011;585:2087-99. doi: 10.1016/j. febslet.2010.08.009.

17. Song SJ, Poliseno L, Song MS, Ala U, Webster K, Ng C, et al.
MicroRNA-antagonism regulates breast cancer stemness and metastasis via TET-family-dependent chromatin remodeling. Cell. 2013;154:311-324. doi: 10.1016/j.cell.2013.06.026.

18. Chiu SC, Chung HY, Cho DY, Chan TM, Liu MC, Huang HM, et al. Therapeutic potential of microRNA let-7: tumor suppression or impeding normal stemness. Cell. 2013 Jul 18;154(2):311324. doi: 10.1016/j.cell.2013.06.026.

19. Kortylewski M, Nechaev S. How to train your dragon: targeted delivery of microRNA to cancer cells in vivo. Mol Ther. 2014:22:1070-1071. doi: 10.1038/mt.2014.73.

20. Al-Hajj M, Wicha MS, Benito-Hernandez A, Morrison SJ, Clarke MF. Prospective identification of tumorigenic breast cancer cells. Proc Natl Acad Sci U S A. 2003;100:3983-3988. doi: 10.1073/pnas.0530291100

21. Landskron G, De la Fuente M, Thuwajit P, Thuwajit C, Hermoso MA. Chronic inflammation and cytokines in the tumor microenvironment. J Immunol Res. 2014;2014:149185. doi: 10.1155/2014/149185.

22. Silver DJ, Sinyuk M, Vogelbaum MA, Ahluwalia MS, Lathia JD. The intersection of cancer, cancer stem cells, and the immune system: therapeutic opportunities. Neuro-oncology. 2016;18(2):153-159.

23. Qi Y, Li R-M, Kong F-M, Li H, Yu J-P, Ren X-B. How do tumor stem cells actively escape from host immunosurveillance? Biochem Biophys Res Commun. 2012;420(4):699-703. doi: 10.1016/j.bbrc.2012.03.086.

24. Casbon AJ, Reynaud D2, Park C, Khuc E, Gan DD, Schepers $\mathrm{K}$, et al. Invasive breast cancer reprograms early myeloid differentiation in the bone marrow to generate immunosuppressive neutrophils. Proc Natl Acad Sci U S A. 2015;112:E566-75. doi: 10.1073/pnas.1424927112.

25. Cabarcas SM, Mathews LA, Farrar WL. The cancer stem cell niche-there goes the neighborhood? Int J Cancer. 2011;129(10):2315-27. doi: 10.1002/ijc.26312.

26. Kitamura T, Qian B-Z, Pollard JW. Immune cell promotion of metastasis. Nat Rev Immunol. 2015;15(2):73-86. doi: 10.1038/ nri3789.

27. Reinhart BJ, Slack FJ, Basson M. The 21-nucleotide let-7 RNA regulates developmental timing in Caenorhabditis elegans. Nature. 2000;403:901.doi: 10.1038/35002607

28. Venkatadri R, Muni T, lyer A, Yakisich J, Azad N. Role of apoptosis-related miRNAs in resveratrol-induced breast cancer cell death. Cell Death Dis. 2016;7:e2104. doi: 10.1038/cddis.

29. Chaterjee M, van Golen KL. Breast cancer stem cells survive periods of farnesyl-transferase inhibitor-induced dormancy by undergoing autophagy. Bone Marrow Res. 2011;2011:362938. doi: 10.1155/2011/362938

30. Harrison H, Farnie G, Howell SJ, Rock RE, Stylianou S, Brennan KR, et al. Regulation of breast cancer stem cell activity by signaling through the Notch 4 receptor. Cancer Res. 2010;70:709-18. doi: 10.1158/0008-5472.CAN-09-1681.

31. Savarese TM, Low HP, Baik I, Strohsnitter WC, Hsieh C-C. Normal breast stem cells, malignant breast stem cells, and the perinatal origin of breast cancer. Cancer Res. 2010;70:709-18. doi: 10.1158/0008-5472.CAN-09-1681. 